\title{
Coordinate Basis Transformation Based Linearisation Method for Ordinary Differential Equations
}

\author{
Danlei Ye ${ }^{1,3}$, Xin Jiang ${ }^{1,3, *}$, Guanying Huo ${ }^{1,3}$, Cheng $\mathrm{Su}^{1,3}$, Zehong $\mathrm{Lu}^{2}$, Bolun Wang ${ }^{1,3}$ and Zhiming Zheng ${ }^{1,3}$ \\ ${ }^{1}$ LMIB and School of Mathematics and Systems Science, Beihang University, Beijing, China \\ ${ }^{2}$ School of Mathematical Science, Peking University, Beijing, China \\ ${ }^{3}$ Beijing Advanced Innovation Center for Big Data and Brain Computing (BDBC), Beihang University, Beijing, China \\ ${ }^{*}$ Corresponding author
}

\begin{abstract}
In this paper, a linearisation method for nonlinear ordinary differential equations based on coordinate basis transformation is proposed. In order to transform a nonlinear equation into a linear one, we introduce a general framework to extract the coordinate basis of the differential system. Actually, by introducing a mapping function predefined, this extraction method could connect each state variable of the new system with the variables in the original one. Further, by using vector space for the closure of the Lie derivative, we can analytically obtain the mapping based on the subspace iteration. We argue that this method might be efficient in analyzing and solving ordinary differential systems of complex nonlinear forms.
\end{abstract}

Keywords- coordinate basis; linearisation; Lie derivative

\section{INTRODUCTION}

Differential equations are generally used in the analysis of complex systems from various fields, including physical dynamics, chemistry, engineering, economics and demographics. It has been developed with calculus, and its theory and method has been one of the key points in the development of modern science and technology. Almost all of the equations that describe the actual problems are non-linear. Along with the need of precise descriptions and solving practical problems, as well as the emergence and improvement of large computer, various problems of solving ordinary differential equation increasingly attract the attention of scientists and engineers. However, the studies of nonlinear equations mostly stay on qualitative analyses and numerical solutions. In 1841, Liouvile proved that the Riccati equation

$$
\frac{d x}{d t}=a(t) x^{2}+b(t) x+c(t)
$$

cannot be solved by the elementary integral method except for some special types. For example, the function $\frac{d x}{d t}=x^{2}+y^{2}$, which is simple in form, cannot be solved through elementary integration. Until 2010, this problem was solved with multiple integral iterative series methods [15]. In the 19th century, some problems in astrophysics and other technical sciences needed to study the local and global nature of solutions of complex differential equations. Since a large number of differential equations could not be solved by the elementary integral method, researchers discussed numerical solutions, the properties of solutions about the structure and characteristics of the differential equations or the distribution of curves defined by the differential equations [3-7].

With the vigorous development of nonlinear scientific research, people not only need to discuss individual solutions of differential equations, but also try to understand the general trend and structure of a class of solutions or all the solutions. For example, the number and stability of the solution of nonlinear equations are studied by branching theory. The branch theory also provides a basis for further study of the corresponding dynamic system.

At present, there is no general solution to the nonlinear equation. As mentioned above, nonlinear ordinary differential equations are difficult to calculate and analyze, and the general mathematical method is invalid for nonlinear normal micro systems. Linear ordinary differential equations, in contrast, are more intuitive to show the relationship between each parameter. Moreover, a relatively complete solution and qualitative analysis method have been developed for the given linear ordinary differential equations. Therefore, we can make breakthroughs in the study of nonlinear ordinary differential equation by transforming nonlinear equations into analytically linear equations $[1,2]$.

Some quasilinearization techniques [8] and linearisation examples that satisfy the local property $[9,13,14]$ was presented and proved. In 1973, Liao Shantao presented the theory of canonical equations [10-12]. The main idea of the theory of canonical equations was to return the vector field of the manifold to the vector field in the Euclidean space. It transformed the properties of the phase diagrams of the ordinary differential system in the manifold transformed into ordinary differential equations in Euclidean space, which was achieved by the activity frame.

The theory of canonical equations is quite complete, but the method is too complicated to carry out. Therefore, we discuss the linearisation problem in another way. In this paper, we focus on the extraction of the coordinate basis, mapping the trajectories of the nonlinear space to the linear space. It is more straightforward of the coordinate basis transformation based linearisation method for ordinary differential equation although it is still in its infancy.

This paper is organized as follows. In Section 2, we introduce some definitions and theorems on differential system and vector space. In Section 3, the linearisation method of coordinate basis 
transformation is showed. In Section 4, we give two examples of the linearisation experiment. We summarize our conclusion and give some expectation in the last section.

\section{RELATED WORK}

In this section we will introduce some definitions and theorems on differential system and vector space, which are prepared for our linearisation method.S

\section{A. Mapping and Similarity on Lipschitz Continuous Vector \\ Field}

Consider a mapping: $\alpha: \mathbb{R}^{k} \rightarrow \mathbb{R}^{l}$. Given a set $S \subseteq \mathbb{R}^{k}$, $\alpha(S)$ is defined by applying $\alpha$ to all the elements of $S$. Similarly, the reflect set is $\alpha^{-1}(T):\{\alpha(s) \in T\}$. Define $\mathscr{S}\left\langle X_{0}, \mathscr{F}, X_{I}\right\rangle$ to be a continuous system on the variate $\vec{X}:\left(x_{1}, \ldots, x_{n}\right)$ and $\mathscr{T}\left\langle Y_{0}, \mathscr{G}, Y_{I}\right\rangle$ to be a continuous system on the variate $\overrightarrow{\mathrm{y}}:\left(y_{1}, \ldots, y_{n}\right)$.

Def.1 (Simulation). $\mathscr{T}$ is thought to simulate $\mathscr{S}$ iff there exists a smooth mapping $\alpha: \mathbb{R}^{n} \rightarrow \mathbb{R}^{m}$, in which:

1. $Y_{0} \supseteq X_{0}$ and $Y_{I} \supseteq X_{I}$.

2. For any trajectory $\tau:[0, T) \rightarrow X_{I}$ in $\mathscr{S}, \alpha \circ \tau$ is the trajectory in $\mathscr{T}$.

A simulation relation means that any time trajectory in $\mathscr{S}$ can be mapped into a trajectory in $\widetilde{T}$ through $\alpha$. However, the inversion may not exist since $\alpha$ do not have to be reversible. In other words, there may be some time trajectories in $\mathscr{T}$ which can not be mapped into by any trajectory in $\mathscr{S}$.

and $\mathscr{T}$ are defined as Lipschitz continuous vector field.

Them.1 $\mathscr{T}$ simulates $\mathscr{S}$ if the following conditions are satisfied:

1. $Y_{0} \supseteq X_{0}$.

2. $Y_{I} \supseteq X_{I}$.

3. $(\alpha(\vec{x}))=J_{\alpha} \cdot \mathscr{F}(\vec{x})$, where $J_{\alpha}$ is the Jacobian:

$$
J_{\alpha}\left(x_{1}, \ldots, x_{n}\right)=\left(\begin{array}{ccc}
\frac{\partial \alpha_{1}}{\partial x_{1}} & \cdots & \frac{\partial \alpha_{1}}{\partial x_{n}} \\
\vdots & \ddots & \vdots \\
\frac{\partial \alpha_{m}}{\partial x_{1}} & \cdots & \frac{\partial \alpha_{m}}{\partial x_{n}}
\end{array}\right)
$$

and $\alpha(\vec{x})=\left(\alpha_{1}(\vec{x}), \ldots, \alpha_{m}(\vec{x})\right), \alpha: \mathbb{R}^{n} \rightarrow \mathbb{R}$.

Proof. Let $\tau$ be a trajectory over $\vec{x}$ of the system $\mathscr{S}$. Note that at any time $t \in[0, T), \frac{d \tau_{x}}{d t}=\mathscr{F}\left(\tau_{x}(t)\right)$

We want to proof that $\tau_{y}(t)=\alpha\left(\tau_{x}(t)\right)$ is a time trajectory in $\mathscr{S}$. Since $\tau_{x}(0) \in X_{0}$, it can be concluded that $\tau_{y}(0)=\alpha\left(\tau_{x}(0)\right) \in Y_{0}$. Since $\tau_{x}(t) \in X_{I}$ is established for any $t \in[0, T)$, then $\tau_{y}(t)=\alpha\left(\tau_{x}(t)\right) \in Y_{I}$. Differentiate $\tau_{y}$, then we achieve

$$
\frac{d \tau_{y}}{d t}=\frac{d \alpha\left(\tau_{x}(t)\right)}{d t}=J_{\alpha} \cdot \mathscr{F}\left(\alpha\left(\tau_{x}(t)\right)\right)=\mathscr{F}\left(\tau_{y}(t)\right)
$$

Therefor $\tau_{y}=\alpha \circ \tau_{x}$ conforms to the dynamical system $\mathscr{T}$. Through Lipschitz continuity of $\mathscr{F}$, it shows that $\tau_{y}$ is the unique trajectory starting from $\alpha \circ \tau_{x}(0)$.

It is important to note that, in normal conditions a trajectory $\tau_{y}(t)=\alpha \circ \tau_{x}(t)$ may exist in a longer time interval than $[0, T)$ over which $\tau_{x}(t)$ is assumed to be defined.

Them. 2 Let $\mathscr{T}$ simulate $\mathscr{P}$ through a mapping $\alpha$. If $Y \supseteq Y_{I}$ is a positive invariant set of $\mathscr{T}$, then $\alpha^{-1}(Y) \cap X_{I}$ is a positive invariant set of $\mathscr{S}$.

Def.2 (Linearizing Coordinate Bases Transformation). Let $\mathscr{S}$ be a nonlinear system. $\alpha$ is a linearizing coordinate bases transformation if it maps every trajectory from $\mathscr{S}$ to a affine system $\mathscr{T}$. In other words, $\alpha$ insures that $\mathscr{S}$ simulates a affine system $\mathscr{T}$.

We search for a mapping $\alpha$ to obtain $\mathscr{T}$ which simulates a given system $\mathscr{S}$ through $\alpha$. In other words, we search for a mapping $\alpha$ that satisfies

$$
J_{\alpha}(\vec{x}) \cdot \mathscr{T}(\vec{x})=A(\vec{x})+\vec{b}
$$

$A, b$ are constant matrices. After finding out the mapping, we can always find out the appropriate initial and invariance conditions for the simulating system $\mathscr{T}$ whose dynamics will be given by $(\vec{y})=A(\vec{y})+\vec{b}$.

Def.3 (Lie Derivative). Let $\alpha(\vec{x})=\left(\alpha_{1}(\vec{x}), \ldots, \alpha_{m}(\vec{x})\right)$ be a smooth mapping $\alpha: \mathbb{R}^{n} \rightarrow \mathbb{R}^{m}$, wherein each $\alpha_{i}: \mathbb{R}^{n} \rightarrow \mathbb{R}$. $\mathscr{L}_{F}\left(\alpha_{i}(\vec{x})\right)=\left(\nabla \alpha_{i}\right) \cdot \mathscr{F}(\vec{x})$ indicates the Lie derivative of the function $\alpha_{i}(\vec{x})$ in the vector field $\mathscr{F}$.

Lemma.1

$$
J_{\alpha}(\vec{x}) \cdot \mathscr{F}(\vec{x})=A(\vec{x})+\vec{b}
$$

Proof. Since Jacobian $J_{\alpha}$ :

$$
J_{\alpha}\left(x_{1}, \ldots x_{n}\right)=\left(\begin{array}{ccc}
\frac{\partial y_{1}}{\partial x_{1}} & \cdots & \frac{\partial y_{1}}{\partial x_{n}} \\
\vdots & \ddots & \vdots \\
\frac{\partial y_{m}}{\partial x_{1}} & \cdots & \frac{\partial y_{m}}{\partial x_{n}}
\end{array}\right)=\left(\begin{array}{c}
\nabla \alpha_{1} \\
\vdots \\
\nabla \alpha_{n}
\end{array}\right)
$$


It can be obtained that

$$
J_{\alpha} \cdot \mathscr{T}=\left(\begin{array}{c}
\left(\nabla \alpha_{1}\right) \cdot \mathscr{T} \\
\vdots \\
\left(\nabla \alpha_{n}\right) \cdot \mathscr{T}
\end{array}\right)=\left(\begin{array}{c}
\mathscr{L}_{F}\left(\alpha_{1}(\vec{x})\right) \\
\vdots \\
\mathscr{L}_{F}\left(\alpha_{m}(\vec{x})\right)
\end{array}\right)
$$

Given functions $\alpha_{1}, \alpha_{2}, \ldots, \alpha_{m}: \mathbb{R}^{n} \rightarrow \mathbb{R}$ and constant equations $1: \mathbb{R}^{n} \rightarrow \mathbb{R}$, we consider the vector space generated by these equations:

$$
\operatorname{Span}\left(1, \alpha_{1}, \ldots, \alpha_{m}\right)=\left\{c_{0} \cdot 1+\sum_{i=1}^{m} c_{i} \alpha_{i} \mid c_{0}, \ldots, c_{m} \in \mathbb{R}\right\}
$$

\section{B. The Closure of Vector Space}

Them.3 (Vector Space Closure Theorem). The vector space is closed under Lie derivative. In other words, a mapping $\alpha:\left(\alpha_{1}, \ldots, \alpha_{m}\right)$ represents a linearizing coordinate bases transformation of a system iff the vector space $V: \operatorname{Span}\left(1, \alpha_{1}, \ldots, \alpha_{m}\right)$ is closure under the calculation of Lie derivative.

For example, $\forall g \in V, \mathscr{L}_{F}(g) \in V$.

Proof. Let $\alpha$ be a linearizing coordinate bases transformation which maps trajectories in $\mathscr{F}$ into trajectories in $\mathscr{Y}$ :

$$
\frac{d \vec{y}}{d t}=A \vec{y}+\vec{b}
$$

Therefor to each $\alpha_{i}$,

$$
\mathscr{L}_{F}\left(\alpha_{i}\right)=b_{i}+\sum_{j} A_{i j} \alpha_{j}
$$

Any element $\beta \in V$ can be written as $\beta=c_{0}+\sum_{k} c_{k} \alpha_{k}$ which is a linear combination of the coordinate bases of the vector space. Using (2.6) we can obtain $\mathscr{L}_{F}(\beta)$ which is a linear combination of $\alpha_{i}$ and 1 . Therefor it can be proofed that $\mathscr{L}_{F}(\beta) \in V$.

On the contrary, if $V$ is closure under the calculate of Lie derivative, then its bases $1, \alpha_{1}, \ldots, \alpha_{m}$ satisfy the condition

$$
\mathscr{L}_{F}\left(\alpha_{i}\right)=b_{i} 1+\sum_{j} a_{i j} \alpha_{j}
$$

From Lemma1,

$$
J_{\alpha} \cdot \mathscr{F}=\left(\begin{array}{c}
\mathscr{L}_{F}\left(\alpha_{1}(\vec{x})\right) \\
\vdots \\
\mathscr{L}_{F}\left(\alpha_{m}(\vec{x})\right)
\end{array}\right)=A \alpha(\vec{x})+\vec{b}
$$

wherein $A=\left[\alpha_{i j}\right], \vec{b}=\left(b_{i}\right)$. All above results demonstrate that $\alpha$ is a linearizing coordinate bases transformation.

\section{COORDINATE BASIS TRANSFORMATION}

In this section, we will present the linearisation method of coordinate basis transformation for ordinary differential equation space.

\section{A. Subspace Iterative Method}

If the linearising method of coordinate bases transformation exists, it could be found out by the closure of vector space under Lie derivative. Our goal is to find out a vector space that consists of some functions, while this space is closed under the action of Lie derivative.

Given a set of functions $B=\left\{f_{1}, \ldots, f_{k}\right\}$, we use $\operatorname{Span}(B)$ to represent the vector established by the functions in $B$.

$$
\operatorname{Span}(B)=\left\{\sum_{j=1}^{k} a_{j} f_{j}(\vec{x}) \mid a_{j} \in \mathbb{R}\right\}
$$

We use a method of subspace iteration as follows:

1. Choose an initial vector space $V_{0}=\operatorname{Span}\left(\left\{\alpha_{1}, \ldots, \alpha_{N}\right\}\right)$.

2. Use $V_{i}$ to define $V_{i+1}$ at each step, wherein $V_{i+1}$ is the subspace of $V_{i}$.

3. Stop the operation when $V_{n+1}=V_{n}$. IF $V_{n}$ is a non-trivial vector space, then we can achieve a non-trivial linear coordinate basis transformation from the basis of $V_{n}$.

At step 1, we consider to choose the initial vector space $V_{0}$. To those ordinary differential equations with polynomial on the right-hand side, usually $V_{0}$ can be made up of all the monomial expressions whose degrees are less than a given value. Each function $f_{i}$ in $V_{0}$ can be written as $\sum_{i} c_{i} \alpha_{i}$, wherein $c_{i}$ is a parameter. $V_{0}$ can also be represented by these functions in it: $V_{0}=\operatorname{Span}\left(\left\{f_{0}, \ldots, f_{k}\right\}\right)$. There are two methods to establish the initial space: one is setting a high degree of the initial bases of $V_{0}$ and then the degrees would reduce with the bases reduced during the iteration. The other one is setting the same degree $N$ of the initial bases as the highest degree of the polynomial on the right-hand side of the ordinary differential equation, and if the initial space was proved to be improper after the iterative computations, then we would reset the initial degree as $N+1$. In some particular situations, the initial space can be fixed. It will be mentioned later in this paper.

At step 2, $V_{i+1}$ is calculated as follows:

$$
V_{i+1}=\mathscr{Y}\left(V_{i}\right)=\left\{f \in V_{i} \mid \mathscr{L}_{F}(f) \in V_{i}\right\}
$$


Lemma.2 $V_{i+1} \subseteq \mathscr{V}\left(V_{i}\right)$ is the subspace of $V_{i}$, if $V_{i}$ is a vector space.

Proof. Suppose that $V_{i}$ is made up of the bases $\left(1, \alpha_{1}, \ldots, \alpha_{k}\right)$, then it is obvious that $V_{i+1} \subseteq \mathscr{V}\left(V_{i}\right)$. Now we want to prove that $V_{i+1}$ is a vector space. First $1 \in V_{i+1}$. Let $f_{1}, \ldots, f_{l}$ are functions in $V_{i+1}$, it can be obtained that $\mathscr{V}\left(f_{1}\right), \ldots, \mathscr{O}\left(f_{l}\right) \in V_{i}$. The affine combination of the functions is $f: a_{0} 1+\sum_{j=1}^{l} a_{j} f_{j}$, wherein $a_{1}, \ldots, a_{1}$. Its Lie derivative is

$$
\mathscr{L}_{F} f\left(a_{0} 1+\sum_{j=1}^{l} a_{j} f_{j}\right)=a_{j} \sum_{j=1}^{l} \mathscr{L}_{F}(f)_{j}
$$

Therefor $\mathscr{L}_{F} f$ can be written as the linear combination of the Lie derivatives of $f_{1}, \ldots, f_{l}$ in $V_{i}$. Thus $\mathscr{L}_{F}(f) \in V$ and then $f \in V_{i+1}$. Each linear combination of the elements in $V_{i+1}$ belongs to $V_{i}$ as well.

Them.4 Given an initial vector space $V_{0}$ and vector field $\mathscr{F}$, the space will be converged to a subspace $V^{*} \subseteq V_{0}$ through limited steps of iteration. Let $\alpha_{1}, \ldots, \alpha_{m}$ be the base functions which establish $V^{*}$,

1. The transformation $\alpha:\left(\alpha_{1}, \ldots, \alpha_{m}\right)$, which is made up of the base function of the space that we obtain finally, is linear.

2. To each linearising coordinate bases transformation $\beta:\left(\beta_{1}, \ldots, \beta_{m}\right), \beta \in V^{*}$ for each $\beta \in V_{0}$.

Proof. If $V_{i+1} \subseteq V_{i}$ is converged through the iteration, then the degree of $V_{i+1}$ would be less than $V_{i}$. Since the degree of $V_{0}$ is limited, the upper limit of the iteration is the same as the number of the base equations of $V_{0}$.

We suppose that there exists a linearisation coordinate basis transformation $\beta:\left(\beta_{1}, \ldots, \beta_{m}\right)$ which satisfied $\beta_{i} \in V_{0}$. Note that the space $U$ established by $1, \beta_{1}, \ldots, \beta_{m}$ is the subset of $V_{0}$. It also can be proved that if $U \subseteq V_{i}$, then $U \subseteq V_{i+1}$. Through the inductive method, $U \subseteq V^{*}$ is proved.

\section{B. The Algorithm}

Suppose the initial ODE: $\dot{X}=F(X)$, wherein $X=\left(x_{1}, \ldots, x_{n}\right)^{T}, F=\left(f_{1}, \ldots, f_{n}\right)^{T}$.

1. Observe the the right-hand side of the equation to get the highest degree $N$. Set non-negative integer parameters $l, m, l_{m}, i, k$.
2. Establish a set

$$
V=\left\{\prod_{\substack{1 \leq m \leq n \\ \sum_{l_{m}=l}}} x_{m}^{l_{m}} \mid 0 \leq l \leq N\right\} \triangleq\left\{y_{k} \mid 1 \leq k \leq M\right\} .
$$

3. Set $f=\sum_{k \in \tau_{V}} c_{k} y_{k}$, wherein $\tau_{k}$ is the set of the indexes of $V$. Calculate the Lie derivative $\mathscr{L}_{F}(f)=\sum_{i=1}^{n}\left(\frac{\partial f}{\partial x_{i}}\right) \cdot f_{i} \cdot$

4. Compare the Lie derivative $\mathscr{L}_{F}(f)$ and the right-hand side of the equation $F(X)$. If an element $y_{k}$ did not exist in $F(X)$ or $V$, let the coefficient of $y_{k}$ in the integrated $\mathscr{L}_{F}(f)$ be zero.

5. Arrange $\mathscr{L}_{F}(f)$ once again. Select the nonzero terms to establish a new set $V_{1}$.

6. If $V_{1}=\Phi$, then we set $N=N+1$ and return to step 2; if $V_{1}=V$, then stop the steps and output $V$; otherwise, set $V=V_{1}$ and return to step 3.

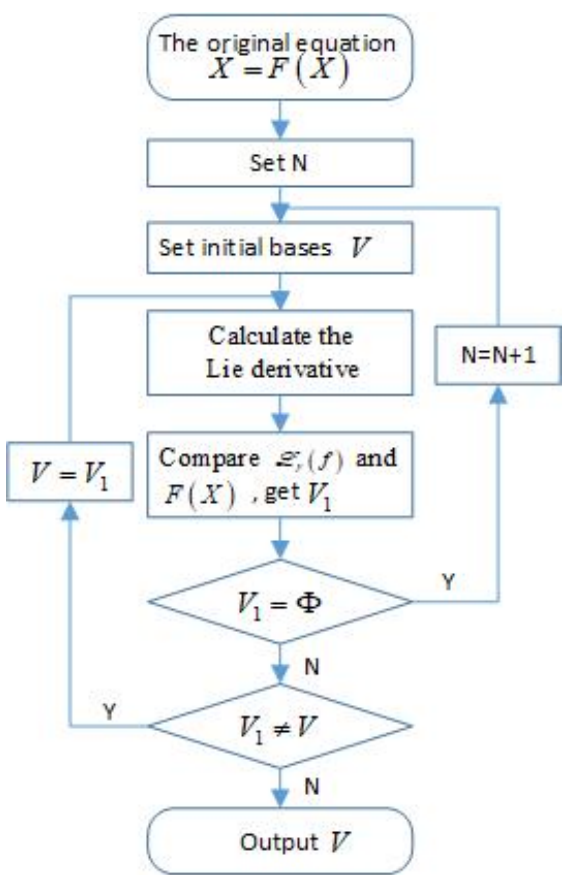

FIGURE I. ALGORITHM FLOW CHART OF THE SUBSPACE ITERATIVE METHOD

\section{The Initial Bases Selection}

It seems to be straightforward to linearising any non-linear ordinary differential equation while the generic algorithm has been applied. Nevertheless, because of the uncertainty of the initial base selection, the iterative algorithm will be quite tedious when the degree of the initial differential system is high. And in the actual engineering problems, most often we just need to analyze systems of ordinary differential equations with three or two elements. 
We found that for a system of three-dimensional ordinary differential equations, when the right-hand side of the equation only includes constant terms, one degree terms and cross terms, it is able to set

$$
\left(x_{1}^{2}, x_{1} x_{2}, x_{1} x_{3}, x_{1}, x_{2}^{2}, x_{2} x_{3}, x_{2}, x_{3}^{2}, x_{3}, 1\right)
$$

as the initial bases. In other words, any three-dimensional ordinary differential equations which only includes constant terms, one degree terms and cross terms in the right-hand side of the equations, can be transformed into linear ordinary differential equations with no more than nine elements. More experiments are shown in the next section.

\section{EXPERIMENT}

In this section, we present two examples of the linearisation method based on coordinate basis transformation.

Exp.1 Given a non-linear ordinary differential equation

$$
\left\{\begin{array}{l}
\dot{x}_{1}=9 x_{1}+3 x_{2}+10 x_{3}+10 x_{1} x_{2}+x_{1} x_{3}+8 x_{2} x_{3}+8 \\
\dot{x}_{2}=6 x_{1}+5 x_{2}+2 x_{3}+5 x_{1} x_{2}+4 x_{1} x_{3}+10 x_{2} x_{3}+9 \\
\dot{x}_{3}=x_{1}+10 x_{2}+10 x_{3}+8 x_{1} x_{2}+9 x_{1} x_{3}+7 x_{2} x_{3}+1
\end{array}\right.
$$

Based on the previous conclusion, we directly set the initial bases $Y=\left(y_{1}, \ldots, y_{9}, 1\right)$ :

$$
\left\{\begin{array}{ccc}
y_{1}=x_{1}^{2} & y_{2}=x_{1} x_{2} & y_{3}=x_{1} x_{3} \\
y_{4}=x_{1} & y_{5}=x_{2}^{2} & y_{6}=x_{2} x_{3} \\
y_{7}=x_{2} & y_{8}=x_{3}^{2} & y_{9}=x_{3}
\end{array}\right.
$$

Then the linearising result shows as follows:

$$
\left\{\begin{array}{l}
\dot{y}_{1}=18 y_{1}+6 y_{2}+20 y_{3}+16 y_{4} \\
\dot{y}_{2}=6 y_{1}+14 y_{2}+2 y_{3}+9 y_{4}+3 y_{5}+10 y_{6}+8 y_{7} \\
\dot{y}_{3}=y_{1}+10 y_{2}+19 y_{3}+y_{4}+3 y_{6}+10 y_{8}+8 y_{9} \\
\dot{y}_{4}=10 y_{2}+y_{3}+9 y_{4}+8 y_{6}+3 y_{7}+10 y_{9}+8 \\
\dot{y}_{5}=12 y_{2}+10 y_{5}+4 y_{6}+18 y_{7} \\
\dot{y}_{6}=y_{2}+6 y_{3}+10 y_{5}+15 y_{6}+y_{7}+2 y_{8}+9 y_{9} \\
\dot{y}_{7}=5 y_{2}+4 y_{3}+6 y_{4}+10 y_{6}+5 y_{7}+2 y_{9}+9 \\
\dot{y}_{8}=2 y_{3}+20 y_{6}+20 y_{8}+2 y_{9} \\
\dot{y}_{9}=8 y_{2}+9 y_{3}+y_{4}+7 y_{6}+10 y_{7}+10 y_{9}+1
\end{array}\right.
$$

The original three-dimensional nonlinear ordinary differential equation is converted to a nine-dimensional linear ordinary differential equation. The other experiment will present that the linearising result of the same kind of nonlinear ordinary differential equation may be more concise.

Exp.2 Given a non-linear ordinary differential equation

$$
\left\{\begin{array}{c}
\dot{x}_{1}=3 x_{1}+x_{2}+4 x_{3}+4 x_{1} x_{2}+7 x_{1} x_{3}+7 x_{2} x_{3}+4 \\
\dot{x}_{2}=10 x_{1}+7 x_{2}+5 x_{3}+10 x_{1} x_{2}+7 x_{1} x_{3}+7 x_{2} x_{3}+2 \\
\dot{x}_{3}=9 x_{1}+3 x_{2}+9 x_{3}+3 x_{1} x_{2}+5 x_{1} x_{3}+2 x_{2} x_{3}+5
\end{array}\right.
$$

We select the same initial baese as in Exp 1 and after the iterative steps, the bases have been simplified:

$$
\left\{\begin{array}{lll}
y_{1}=x_{1} x_{2} & y_{2}=x_{1} x_{3} & y_{3}=x_{2} x_{3} \\
y_{4}=x_{1} & y_{5}=x_{2} & y_{6}=x_{3}
\end{array}\right.
$$

Then the linearising result is:

$$
\left\{\begin{array}{l}
y_{1}=10 y_{1}+5 y_{2}+2 y_{3}+4 y_{4}+4 y_{5} \\
y_{2}=3 y_{1}+12 y_{2}+5 y_{3}+y_{4}+4 y_{6} \\
y_{3}=4 y_{1}+7 y_{2}+3 y_{3}+7 y_{4}+y_{5}+4 y_{6}+4 \\
y_{4}=9 y_{1}+10 y_{2}+16 y_{4}+5 y_{5}+2 y_{6} \\
y_{5}=10 y_{1}+7 y_{2}+10 y_{3}+7 y_{4}+7 y_{5}+5 y_{6}+2 \\
y_{6}=3 y_{1}+5 y_{2}+9 y_{3}+2 y_{4}+3 y_{5}+9 y_{6}+5
\end{array}\right.
$$

\section{CONCLUSION AND EXPECTATION}

In this paper, we demonstrate that in most cases there exist a linearising coordinate bases transformation which could map a given non-linear ordinary differential system into a linear one. It is shown that the coordinate bases can be obtained through limited iteration steps. It is interesting to find that if a threedimensional ordinary differential system only includes constant terms, one degree terms and cross terms, it can be always transformed into a linear ordinary differential system with no more than nine term. We argue that this linearising algorithm is exercisable in case of given appropriate initial bases. However, searching for the affine transformation of multivariate highdimensional non-linear ordinary differential equations is always tedious. We might investigate this problem in our future studies.

\section{REFERENCES}

[1] Sankaranarayanan S. Automatic abstraction of non-linear systems using change of bases transformations[C]. ACM International Conference on Hybrid Systems: Computation and Control, HSCC 2011, Chicago, Il, Usa, April. DBLP, 2011:143-152

[2] Sankaranarayanan S. Change-of-bases abstractions for non-linear hybrid systems[J]. Nonlinear Analysis Hybrid Systems, 2016, 19:107-133.

[3] Harald M, nch. Boundary value problems for nonlinear ordinary differential equations of second order in Banach spaces[J]. Nonlinear Analysis Theory Methods \& Applications, 1984, 8(12):1481-1487.

[4] Rebiha R, Matringe N, Moura A V. Transcendental inductive invariants generation for non-linear differential and hybrid systems[C]. 2012:25-34.

[5] Hairer E, Norsett S P, Wanner G. Solving Ordinary Differential Equations I[J]. Bulletin Des Sciences Mathematiques, 1993, 137(2):189-214.

[6] Hairer E, Wanner G. Solving Ordinary Differential Equations II[J]. Bulletin Des Sciences Mathematiques, 1980, 137(2):189-214.

[7] Butcher J C. The Numerical Analysis of Ordinary Differential Equations, Runge-Kutta and General Linear Methods[J]. Mathematics of Computation, 1987, 51(183):693-693.

[8] Baird C A. Modified quasilinearization technique for the solution of boundary-value problems for ordinary differential equations[J]. Journal of Optimization Theory \& Applications, 1969, 3(4):227-242. 
[9] Ramos J I. Linearization techniques for singularly-perturbed initial-value problems of ordinary differential equations[J]. Applied Mathematics \& Computation, 2005, 161(2):525-542.

[10] Liao S. Qualitative theory of differential dynamic system[M]. Science Publishing House, 1992.

[11] Liao S. Linearization and canonical equations[J]. The practice and cognition of mathematics, 1973(2):30-35.

[12] Liao S. Canonical equations[J]. Journal of mathematics, 1974, 17(2):100109.

[13] Hartman P. On the local linearization of differential equations[J]. Proceedings of the American Mathematical Society, 1963, 14(4):568-573.

[14] Pugh C C. On a Theorem of P. Hartman[J]. American Journal of Mathematics, 1969, 91(2):363-367.

[15] Lin W. Modern mathematics and quantum mechanics[M]. Zhengzhou University Press, 2010. 